\title{
Case Report: Incidental Finding of COVID-19 Infection after Positron Emission Tomography/CT Imaging in a Patient with a Diagnosis of Histoplasmosis and Recurring Fever
}

\author{
Camila Edith Stachera Stasiak, ${ }^{1}$ David Henrique Nigri, ${ }^{2}$ Fabrícius Rocha Cardoso, ${ }^{1}$ \\ Raphael Santos de Almeida Rezende de Mattos, ${ }^{3}$ Philippe Alcântara Gonçalves Martins, ${ }^{3}$ Alysson Roncally Silva Carvalho, ${ }^{4,5,6}$ \\ Sérgio Altino de Almeida, ${ }^{1}$ Rosana Souza Rodrigues, ${ }^{3}$ and Paulo Henrique Rosado-de-Castro ${ }^{1,3,7 *}$ \\ ${ }^{1}$ Department of Radiology, D'Or Institute for Research and Education, Botafogo, Rio de Janeiro, Brazil; '2 Samaritano Hospital, Rio de Janeiro, Brazil; \\ ${ }^{3}$ Department of Radiology, Federal University of Rio de Janeiro, Rio de Janeiro, Brazil; ${ }^{4}$ Cardiovascular R\&D Centre (UnIC), Department of Surgery \\ and Physiology, Faculty of Medicine, University of Porto, Porto, Portugal; ${ }^{5}$ Laboratory of Pulmonary Engineering, Biomedical Engineering Program, \\ Alberto Luiz Coimbra Institute of Post-Graduation, Research in Engineering, Universidade Federal do Rio de Janeiro, Rio de Janeiro, Brazil; \\ ${ }^{6}$ Laboratory of Respiration Physiology, Carlos Chagas Filho Institute of Biophysics, Universidade Federal do Rio de Janeiro, Rio de Janeiro, Brazil; \\ ${ }^{7}$ Institute of Biomedical Sciences, Federal University of Rio de Janeiro, Rio de Janeiro, Brazil
}

\begin{abstract}
This is a case report of a 37-year-old woman evaluated with 18F-fludeoxyglucose (18F-FDG) positron emission computed tomography/CT with recurrent fever after treatment with itraconazole for 6 weeks for histoplasmosis. The examination demonstrated a decrease in the dimensions of the pulmonary opacities previously identified in the left lower lobe and attributed to histoplasmosis. In addition to these pulmonary opacities, increased FDG uptake was also observed in lymph nodes present in the cervical region, mediastinum, left lung hilum, and hepatic hilum. Notably, other pulmonary opacities with ground-glass pattern that were not present in the previous computed tomography were detected in the right lower lobe, with mild 18F-FDG uptake. Nasal swab performed shortly after the examination was positive for COVID-19. In this case, the 18F-FDG positron emission computed tomography/CT study demonstrated findings consistent with active COVID-19 infection coexisting with inflammatory changes associated with histoplasmosis infection.
\end{abstract}

\section{CASE REPORT}

A 37-year-old woman with no comorbidities from Rio de Janeiro presented with mild afternoon fever, night sweating, and chest pain in February 2020 for 12 days. On February 13, 2020, a thorax computed tomography (CT) was requested and showed two irregular pulmonary opacities with central necrosis localized paracardially in the anteromedial basal segment of the inferior lobe in the left lung, the larger one measuring $2.2 \times 2.0 \mathrm{~cm}$, in addition to lymph nodes in the left hilum and minor pleural effusion in the same lung. She underwent investigation with several tests. Interferon-gamma release assay was negative, and the $\mathrm{M}$ band was positive for histoplasmosis in the immunodiffusion assay. She did not have a recent history of international travel. She started the treatment with itraconazole on February 19. The patient had a slow recovery, with recurrent fatigue and malaise. Six weeks after the beginning of the treatment, she presented recurring mild fever, fatigue, dyspnea, and chest pain. Her attending physician requested an 18F-fludeoxyglucose (18F-FDG) PET/CT for differential diagnosis between active histoplasmosis and lymphoma. The PET/CT was performed on April 1, 2020 and compared with the previous CT from February 13, 2020 (Figures 1, 2, and 3). A decrease in the dimensions of the pulmonary opacities previously identified in the left lower lobe was noted, but these opacities had significant radiotracer uptake (SUVmax 6.0). Positive lymph nodes were present in the cervical region (level Ila, bilaterally), mediastinum (subcarinal), left lung hilum, and hepatic hilum. These findings were consistent with inflammatory findings associated with histoplasmosis infection. Notably, other pulmonary opacities with ground-

*Address correspondence to Paulo Henrique Rosado-de-Castro, D'Or Institute for Research and Education, Rua Diniz Cordeiro 30, Botafogo, 22281-100, Rio de Janeiro/RJ, Brazil. E-mail: paulo.rosado@idor.org glass pattern that were not present in the previous CT were detected in the right lower lobe, with mild 18F-FDG uptake-SUVmax 2.5. These findings were highly suggestive for COVID-19. The attending physician and patient were informed of the possibility of COVID-19 infection, and the patient underwent an RT-PCR through nasal swab, which was positive for COVID-19. She observed the social distancing measures and was followed up by her assistant physician. The patient did not present severe symptoms and did not require hospitalization. She repeated the thorax CT on May, 18th for disease control. It showed resolution of the pulmonary opacities with ground-glass pattern in the right lower lobe. The irregular pulmonary opacities in the anteromedial basal segment of the inferior lobe in the left lung were still present but had additional decrease in its dimensions. The left hilum and subcarinal lymph nodes presented decrease in its dimensions as well. The minor pleural effusion in the left lung was not observed anymore. She has not repeated positron emission computed tomography/CT (PET-CT) until the present date.

\section{DISCUSSION}

In December 2019, a novel coronavirus (SARS-CoV-2) emerged in Wuhan, China, later becoming a pandemic. Brazil experienced a significant rise in the number of patients with severe acute respiratory illness in the first 12 epidemiological weeks of 2020 , higher than that in the past 10 years, which is attributed to be due to the new COVID-19 infection. ${ }^{1,2}$ The first COVID-19 Brazilian case was confirmed on February 25, 2020. ${ }^{1}$ Classically, COVID-19 symptoms include fever, cough, fatigue, dyspnea, and headache, but many patients could be asymptomatic. 3,4

Computed tomography and $18 \mathrm{~F}$-fludeoxyglucose (18FFDG) PET-CT findings in suspected or confirmed COVID19 infection have been described in different reports. ${ }^{5-11}$ 


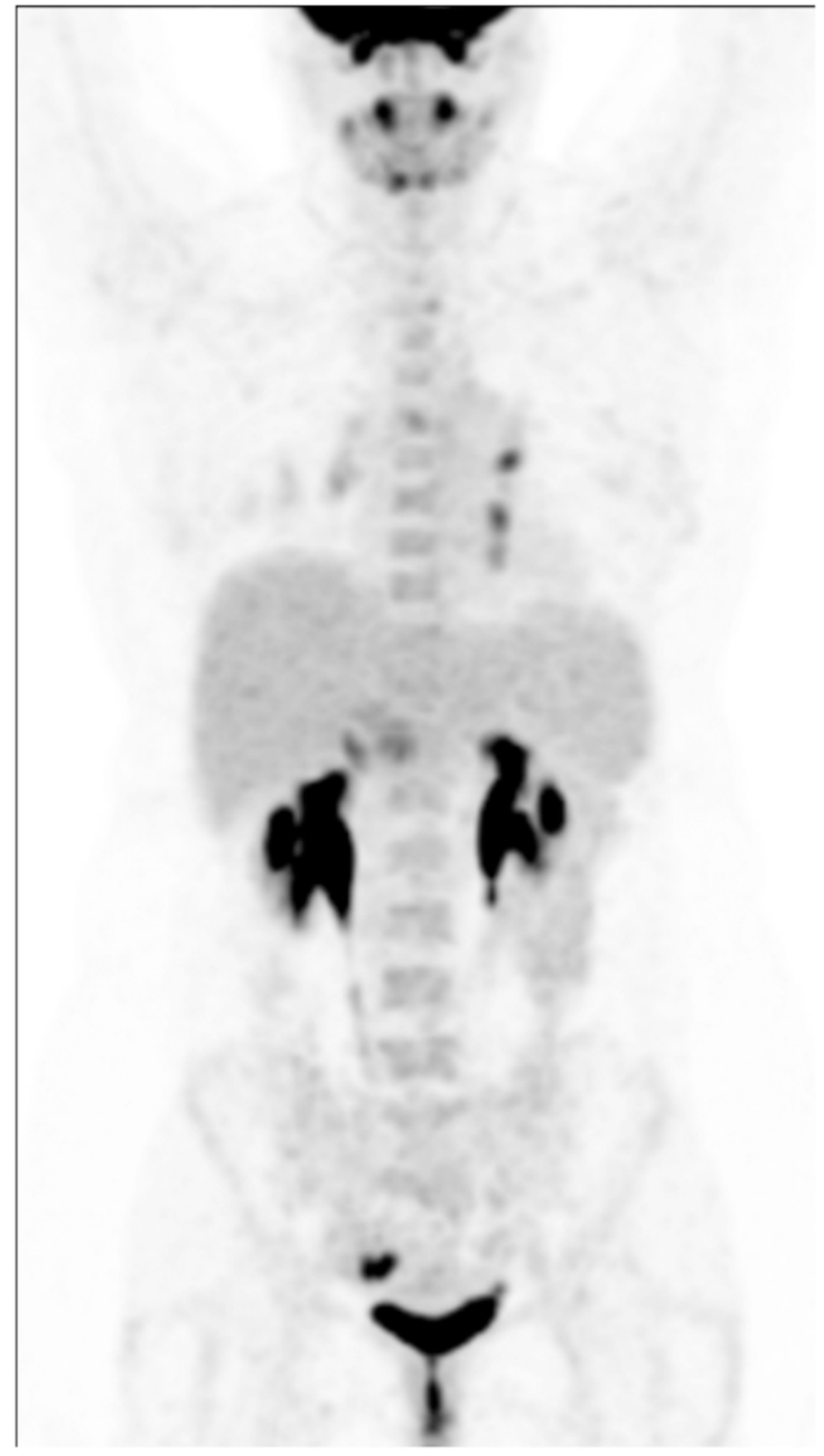

FIGURE 1. Maximum intensity projection image of position emission tomography.

Multiple areas in both lungs could present ground-glass opacities, crazy-paving patterns, consolidations, or linear opacities. Air bronchogram, bronchiectasis, and pleural effusion are less frequent. The opacities tend to occur mainly in peripheral regions in both lungs, mainly in the lower lobe. These abnormalities do not reflect active inflammation, because it could persist a long time after the resolution of the symptoms. ${ }^{12}$

Chest CT is not able to differentiate active inflammation from residual abnormalities. It is not defined yet on how long it could take to the patient to present a normal chest CT after COVID-19 infection. On the other hand, PET-CT is able to show active inflammation and/or infection. PET-CT could differentiate active disease from scar tissue and other residual processes.

Generally, the ground-glass opacities and/or consolidations at CT are 18F-FDG avid at PET-CT.7-11 It is well established that inflammatory processes could present $18 \mathrm{~F}-$ FDG uptake, mainly caused by an increase in glucose consumption. FDG uptake is increased in active inflammation, mainly because of the anaerobic glycolysis seen in inflammatory cells, such as mononuclear cells. ${ }^{13}$ FDG uptake also increases in the inflammatory site because of cytokines and released growth factors. A high cellular metabolic rate generates high FDG uptake. ${ }^{14}$ Therefore, this metabolic pattern cannot distinguish between COVID and other inflammatory or infectious diseases, such as histoplasmosis. Although enlargement of lymph nodes is not described in $\mathrm{CT}$, lymph nodes may have high radiotracer uptake, even in early phases of SARS-CoV-2 infection. ${ }^{7,14}$ It is described that mild nasopharyngeal, bone marrow, and splenic increased FDG uptake has also been described, probably related to the inflammatory condition. ${ }^{13}$ The intensity of FDG uptake can be variable depending on the phase of the disease. ${ }^{15}$ The intensity of radiotracer uptake is described in a case report to be mild in the early phase of COVID-19 and higher in the acute severe and resolution phases. ${ }^{16}$ However, more studies are necessary to analyze the pattern of the radiotracer uptake in the different phases of COVID-19 infection.

Furthermore, at the moment, there is no evidence that higher FDG avidity is related to gravity of COVID-19 infection, with hospitalization rate, nor with severe symptoms. Positron emission computed tomography/CT is not proved to be able to detect superinfection from SARS-CoV-2. Clinical parameters remain the gold standard to define the severity of the disease, although the quantitative analysis on the chest CT could be helpful.

Histoplasmosis is a granulomatous disease caused by inhalation of mycelium spores, and primarily affects the lungs and the immune system. This is more likely to take place in areas inhabited by bats or with high rates of bird droppings. Histoplasmosis is endemic in severalregions of the world, especially in the United States and in parts of Latin America. ${ }^{17}$ In Brazil, it could be found in all regions, but Rio de Janeiro is the state with most of the cases. ${ }^{18}$ Histoplasmosis may present with pulmonary and lymph node involvement, but hematogenic dissemination may occur in any organ or system. ${ }^{18}$ Immunocompetent patients could be asymptomatic. Symptoms include pallor, cough, fever, headache, enlargement of superficial lymph nodes, and hepatosplenomegaly. There have been reports on the 18F-FDG PET/CT findings in histoplasmosis, where lesions are FDG-avid. ${ }^{19-21}$ In addition to the management of lymphoma and other malignancies, 18F-FDG PET-CT may be useful in cases of fungal infections ${ }^{22}$ and in fever of unknown origin. ${ }^{23}$

It is extremely important that assistant physicians and imaging services are aware of the possibility of COVID-19 infection in the differential diagnosis and the possibility of coinfection with other pulmonary etiologies, taking appropriate measures after the identification of a suspicious case. In the described case, when the patient presented with recurring mild fever, fatigue, dyspnea, and chest pain, COVID-19 should have been in the differential diagnosis, and PCR for SARS-CoV-2 should have been performed before PET-CT. Attending physicians should have a low threshold to suspect COVID-19, taking into account the current epidemiologic moment. Recommendations have also been published concerning the standardized reporting of radiological findings and the 

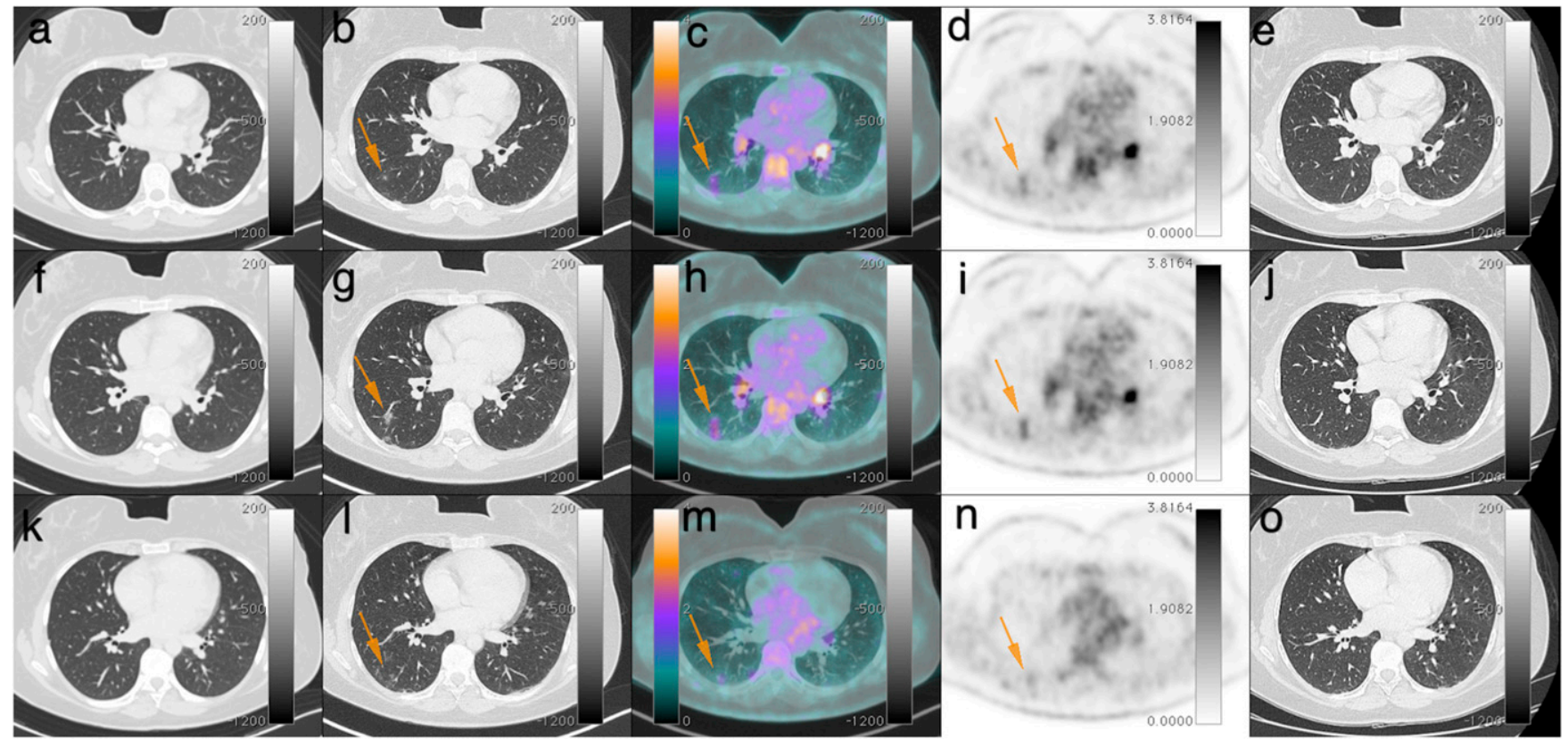

FiguRE 2. Axial images of thorax computed tomography (CT) from February 13, 2020 (A, F, and $\mathbf{K}$ ), axial thorax CT (B, G, and L), axial positron emission computed tomography/CT (PET-CT) fusion (C, H, and $\mathbf{M}$ ), and axial PET (D, I, and $\mathbf{N}$ ) from April 1, 2020, and axial thorax CT from May 18, 2020 (E, J, and O). PET-CT showed pulmonary opacities with ground-glass pattern in the right lower lobe that were not present in the previous CT, with mild 18F-FDG uptake. The last CT showed resolution of the opacities. This figure appears in color at www.ajtmh.org.

use of chest imaging in management during the COVID-19 pandemic, and should be considered when performing PET-CT studies. ${ }^{24,25}$

The present report is relevant to alert doctors that even if the patient had one disease like histoplasmosis and after treatment he or she starts to present the same symptoms present previously, it does not mean that the same disease is recurring. It is very important to consider differential diagnosis during the clinical reasoning. COVID-19 is a new disease that could present with different symptoms and should be
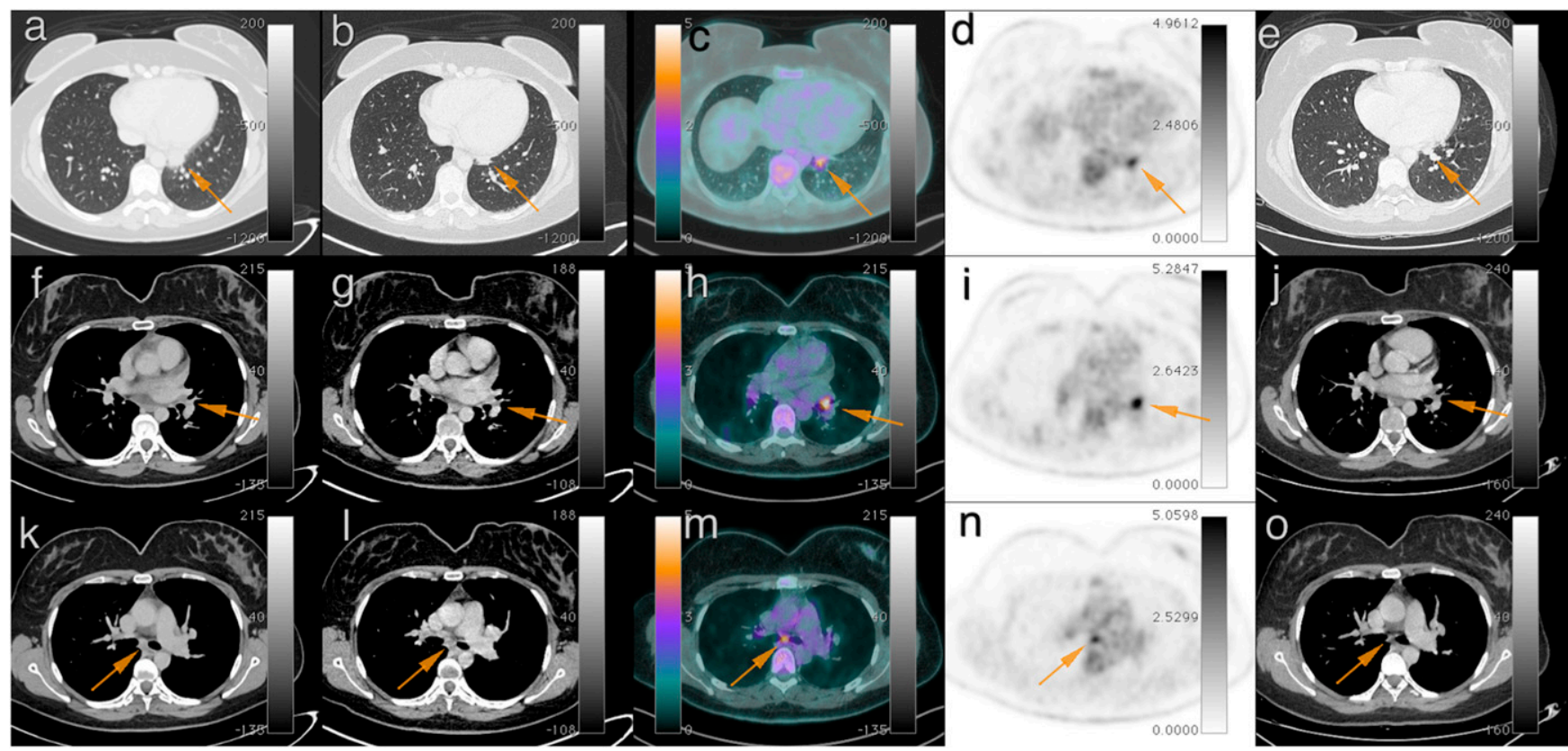

Figure 3. Axial images of thorax computed tomography (CT) from February 13, 2020 (A, F, and K), axial thorax CT (B, G, and L), axial positron emission computed tomography/CT (PET-CT) fusion (C, H, and $\mathbf{M}$ ), and axial PET (D, I, and N) from April 1, 2020, and axial thorax CT from May 18, 2020 (E, J, and O). The first thorax CT shows an irregular pulmonary opacity localized at the anteromedial basal segment in the inferior lobe at the left lung in paracardiac situation, measuring $2.2 \times 2.0 \mathrm{~cm}(\mathbf{A})$. The diameters of this opacity reduced to $1.4 \times 1.3 \mathrm{~cm}(\mathrm{~B})$, but it had significant radiotracer uptake with SUVmax 6.0 in the PET-CT (C and D). Left hilum ( $\mathbf{F}$ and $\mathbf{G})$ and subcarinal (K and $\mathbf{L})$ lymph nodes with radiotracer uptake (H, I, $\mathbf{M}$, and $\mathbf{N}$ ) were unchanged in size but were FDG-avid. The dimensions of the paracardiac opacity (E) and the lymph nodes $(\mathbf{J}$ and $\mathbf{O})$ reduced in the last $\mathbf{C T}$. This figure appears in color at www.ajtmh.org. 
considered. This is the first report in our knowledge that relates COVID-19 and histoplasmosis findings on PET-CT. There is one article in the literature that discusses COVID-19 associated with AIDS-related disseminated histoplasmosis, including CT findings. ${ }^{26}$

Received August 4, 2020. Accepted for publication February 28, 2021. Published online April 2, 2021.

Acknowledgment: Publication charges for this article were waived due to the ongoing pandemic of COVID-19.

Authors' addresses: Camila Edith Stachera Stasiak, Fabrícius Rocha Cardoso, Sergio Altino de Almeida, D'Or Institute for Research and Education, Radiology, Rio de Janeiro, Brazil, E-mails: camilastasiak@ gmail.com, briciusrocha@gmail.com, and altino.sergio@gmail.com. David Henrique Nigri, Samaritano Hospital, Rio de Janeiro, Brazil, E-mail: davidhnigri@globo.com. Raphael Santos de Almeida Rezende de Mattos, Philippe Alcântara Gonçalves Martins, Rosana Souza Rodrigues, and Paulo Henrique Rosado-de-Castro, Department of Radiology, Federal University of Rio de Janeiro, Rio de Janeiro, Brazil, E-mails: raphaelmattos99@gmail.com, philippe.martins19@gmail.com, rosana.rodrigues@idor.org, and paulo.rosado@idor.org. Alysson Roncally Carvalho, Carlos Chagas Filho Institute of Biophysics, Federal University of Rio de Janeiro, Rio de Janeiro, Brazil, E-mail: acarvalho@ biof.ufrj.br.

This is an open-access article distributed under the terms of the Creative Commons Attribution (CC-BY) License, which permits unrestricted use, distribution, and reproduction in any medium, provided the original author and source are credited.

\section{REFERENCES}

1. Cimerman S, Chebabo A, Cunha CAD, Rodriguez-Morales AJ, 2020. Deep impact of COVID-19 in the healthcare of Latin America: the case of Brazil. Braz J Infect Dis 24: 93-95.

2. Bastos LS, Niquini RP, Lana RM, Villela DAM, Cruz OG, Coelho FC, Codeco CT, Gomes MFC, 2020. COVID-19 and hospitalizations for SARI in Brazil: a comparison up to the 12th epidemiological week of 2020. Cad Saude Publica 36: e00070120.

3. Yoshimura Y, Sasaki H, Horiuchi H, Miyata N, Tachikawa N, 2020. Clinical characteristics of the coronavirus disease 2019 (COVID-19) outbreak on a cruise ship. J Infect Chemother 26: 1177-1180.

4. Tian S et al., 2020. Characteristics of COVID-19 infection in Beijing. J Infect 80: 401-406.

5. Zou S, Zhu X, 2020. FDG PET/CT of COVID-19. Radiology 296: E118.

6. Setti L, Kirienko M, Dalto SC, Bonacina M, Bombardieri E, 2020. FDG-PET/CT findings highly suspicious for COVID-19 in an Italian case series of asymptomatic patients. Eur J Nucl Med Mol Imaging 47: 1649-1656.

7. Qin C, Liu F, Yen TC, Lan X, 2020. (18)F-FDG PET/CT findings of COVID-19: a series of four highly suspected cases. Eur J NuCl Med Mol Imaging 47: 1281-1286.

8. Liu C, Zhou J, Xia L, Cheng X, Lu D, 2020. 18F-FDG PET/CT and serial chest CT findings in a COVID-19 patient with dynamic clinical characteristics in different period. Clin Nucl Med 45: 495-469.
9. Kirienko M, Padovano B, Serafini G, Marchiano A, Gronchi A, Seregni E, Alessi A, 2020. CT, [(18)F]FDG-PET/CT and clinical findings before and during early COVID-19 onset in a patient affected by vascular tumour. Eur $\mathrm{J} \mathrm{Nucl} \mathrm{Med} \mathrm{Mol} \mathrm{Imaging} \mathrm{47:}$ 1769-1770.

10. Amini H, Divband G, Montahaei Z, Dehghani T, Kaviani H, Adinehpour Z, Akbarian Aghdam R, Rezaee A, Vali R, 2020. A case of COVID-19 lung infection first detected by [18F]FDG PET-CT. Eur J Nucl Med Mol Imaging 47: 1771-1772.

11. Albano D et al., 2020. Incidental findings suggestive of COVID-19 in asymptomatic patients undergoing nuclear medicine procedures in a high prevalence region. $J$ Nucl Med 61: 632-636.

12. Ding X, Xu J, Zhou J, Long Q, 2020. Chest CT findings of COVID19 pneumonia by duration of symptoms. Eur J Radiol 127: 109009.

13. Dietz M et al., 2020. COVID-19 pneumonia: relationship between inflammation assessed by whole-body FDG PET/CT and shortterm clinical outcome. Eur J Nucl Med Mol Imaging 48: 260-268.

14. Cosma L, Sollaku S, Frantellizzi V, De Vincentis G, 2020. Early 18F-FDG PET/CT in COVID-19. J Med Imaging Radiat Oncol 64: 671-673.

15. Fu C, Zhang W, Li H, Bai Y, Bae KT, Wang M, Xu J, Shao F, 2020. FDG PET/CT evaluation of a patient recovering from COVID-19. Eur J Nucl Med Mol Imaging 47: 2703-2705.

16. Ajuria-Illarramendi O, Martinez-Lorca A, Orduña-Diez MDP, 2020. [18F]FDG-PET/CT in different COVID-19 phases. IDCases 21: e00869.

17. Wheat LJ, Azar MM, Bahr NC, Spec A, Relich RF, Hage C, 2016. Histoplasmosis. Infect Dis Clin North Am 30: 207-227.

18. Aide MA, 2009. Chapter 4--histoplasmosis. J Bras Pneumol 35: $1145-1151$.

19. Kadaria D, Archie DS, SultanAli I, Weiman DS, Freire AX, Zaman MK, 2013. Dual time point positron emission tomography/ computed tomography scan in evaluation of intrathoracic lesions in an area endemic for histoplasmosis and with high prevalence of sarcoidosis. Am J Med Sci 346: 358-362.

20. Kooblall M, Keane B, Murray G, Moloney E, 2014. Histoplasmosis mimicking primary lung neoplasm. BMJ Case Rep 2014: bcr2013203335.

21. Montagnac C, Eldin C, Thouret A, Ranque S, Brouqui P, 2019. Histoplamosis in an immunocompetent man returning from Brazil: a diagnostic challenge helped by 18 FDG PET CT. Travel Med Infect Dis 27: 136-138.

22. Sharma P, Mukherjee A, Karunanithi S, Bal C, Kumar R, 2014. Potential role of 18F-FDG PET/CT in patients with fungal infections. AJR Am J Roentgenol 203: 180-189.

23. Hess S, 2020. FDG-PET/CT in fever of unknown origin, bacteremia, and febrile neutropenia. PET Clin 15: 175-185.

24. Rubin GD et al., 2020. The role of chest imaging in patient management during the COVID-19 pandemic: a multinational consensus statement from the Fleischner Society. Chest 158: 106-116.

25. Prokop M et al., 2020. CO-RADS-a categorical CT assessment scheme for patients with suspected COVID-19: definition and evaluation. Radiology 296: 201473.

26. Bertolini M, Mutti MF, Barletta JA, Falak A, Cuatz D, Sisto A, Ragusa MA, Fernandez Claros NO, Rolón MJ, 2020. COVID-19 associated with AIDS-related disseminated histoplasmosis: a case report. Int J STD AIDS 31: 1222-1224. 